\title{
FARMERS' INFORMATION USAGE INTENTION IN CHINA BASED ON THE TECHNOLOGY ACCEPTANCE MODEL
}

\author{
Jingjing Zhang ${ }^{1,2}$,Xiaoshuan Zhang ${ }^{2}$,Weisong $\mathrm{Mu}^{2}$,Jian Zhang ${ }^{2}$, \\ Zetian $\mathrm{Fu}^{2, *}$ \\ ${ }^{1}$ College of Economics \& Management,China Agricultural University,Beijing,P.R.China \\ 100083 \\ ${ }^{2}$ Key laboratory for modern precision agricultural integration Ministry of Education, China \\ Agricultural University, Beijing,P.R.China 100083 \\ *Corresponding author,Address:Key laboratory for modern precision agricultural integration \\ Ministry of Education, China Agricultural University,17 Tsinghua East Road, Beijin, \\ 100083,P.R.China.Tel:+86-10-62736717,Fax:+86-10-62736717,Email: \\ zhangjingjing1982@cau.edu.cn
}

Abstract: Information technology acceptance has received much attention, but little research has been conducted to assess farmers' information adoption. Despite the importance of information, its value will not be realized if farmers are reluctant to accept it. This research aims to study farmers' information adoption in China, in order to provide some decision-making advice for the people and organization who supply the agriculture information. The model of information usage intention has been established based on the Technology Acceptance Model (TAM). A sample of 231 farmers participated in this study. The results show that the factors which influence the usage willingness for information are perceived usefulness, perceived ease of use, learning intention, risk preference and experience in information before. In addition, income and education may also affect the decision.

Keywords: information demand, usage intention, technology acceptance model, perceived usefulness, perceived ease of use, perceived risk

Please use the following format when citing this chapter:

Zhang, J., Zhang, X., Mu, W., Zhang, J. and Fu, Z., 2009, in IFIP International Federation for Information Processing, Volume 295, Computer and Computing Technologies in Agriculture II, Volume 3, eds. D. Li, Z. Chunjiang, (Boston: Springer), pp. 1845-1853. 


\section{INTRODUCTION}

Information is a key factor and has been proved to be significant in the agriculture production and lives in recent years. However, there exits a gap between agriculture information supply and acceptance. Previous studies have been mostly focused on users' acceptance of information technology. The issue about whether farmers are willing to accept information has not been widely discussed.

To address this issue, this study seeks to provide insights into farmers' information usage intention in China. In previous studies, the technology acceptance model (TAM) has been widely used in order to gain a better understanding of information technology adoption. In this study, TAM is put forth to the topic and modifications are made to it. Perceived risk is added to the original model according to characters of farmers and agriculture information service. Perceived risk sometimes plays an important part in consumers' decisions. If farmers consider that information will bring them help with little risk, the information usage intention is great. The aim of this paper is to define the key factors impacting farmers' information adoption.

\section{THE TAM MODEL}

In our research, the TAM model is selected for the main theoretical method.TAM, which originally developed by Davis, has the advantage of being well grounded in established social psychology theory (McKechnie et al.,2006). In the model, two main aspects constitute the model frame: perceived usefulness and perceived ease of use. The former refers to the users' perception of the degree to which using a system will enhance their performance, and the latter means the degree to which users consider the system to be free of effort (Davis et al., 1989).

In the model,perceived usefulness and perceived ease of use affect actual system usage through attitude and intention. Attitude,which is the key determinant of behavioral intention has a direct effect on intention, is determined by both users' perception of usefulness and ease of use, and the actual use is directly determined by behavioral intention. In previous research, it was found that usefulness may impact actual use irrespective of attitude, provided that the use of the system offers direct benefits to the user (Davis et al., 1989; McKechnie et al.,2006). If a system is considered to be easy to master, the perception of the usefulness will be reinforced. Therefore, perceived ease of use may have a direct influence on perceived usefulness. Fig. 1 is the TAM model. 


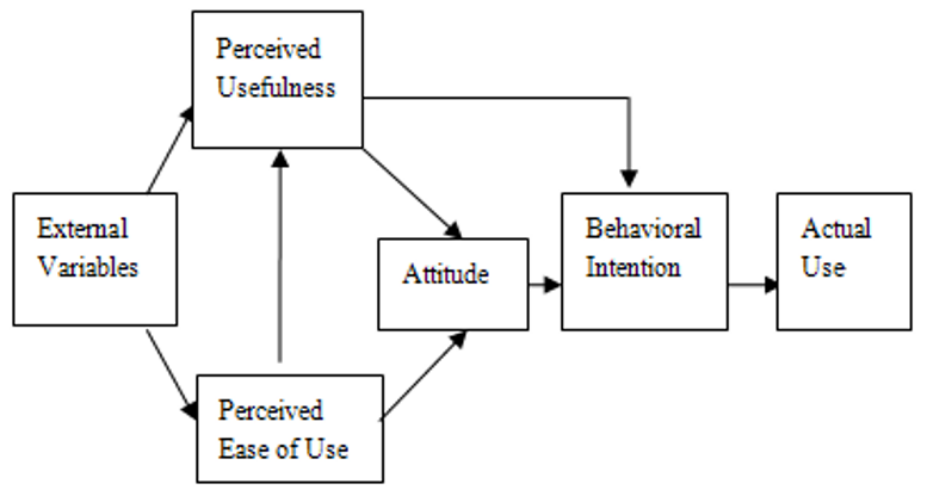

Fig.1: The TAM model

\section{METHODOLOGY}

In this paper, modifications are made to the original model and several hypotheses are put forward based on the model. Fig.2 is the farmer information usage intention model.

\subsection{Factors of the original model}

According to the TAM model, perceived usefulness and perceived ease of use are two important determinants.In this study,it is supposed that perceived usefulness and perceived ease of use affect farmers' attitude directly. Furthermore, it is proposed that perceived ease of use not only predicts attitude, but is also an antecedent of perceived usefulness (Davis et al.,1989). Farmers' confidence in information will be increased if it is easy for them to make use of agriculture information.

H1.Farmers' perceived usefulness influences the attitude towards using information positively.

H2.Farmers' perceived ease of use influences the attitude towards using information positively.

H3.Farmers' perceived ease of use influences perceived usefulness positively.

As is the case with the TAM, attitude towards using information may impact information usage intention directly.Similarly,it is assumed that perceived usefulness affect information intention to use without the medium of attitude (Davis et al.,1989). The next two hypotheses are proposed:

H4.Farmers' perceived usefulness affects the information usage intention positively.

H5.Farmers' attitude affects the usage intention positively. 


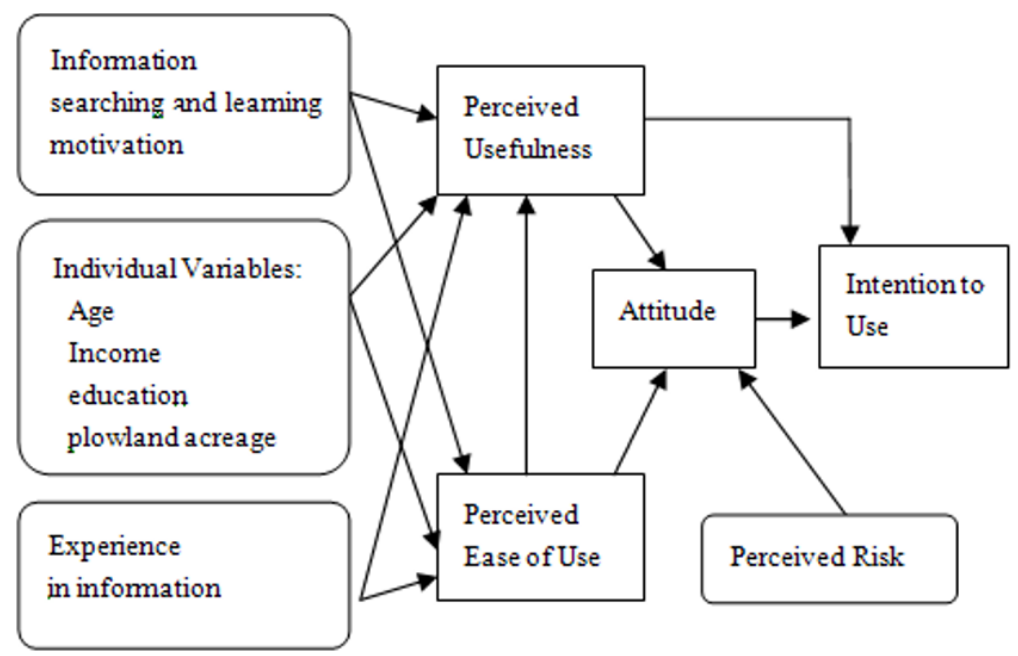

Fig.2: The information usage intention model

\subsection{Experience in agriculture information}

Prior work has suggested that past experience with the technology was a paramout factor and resulted in the higher degree of the adoption (McKechnie et al.,2006). It is argued that farmers who have experience in using information may have stronger usage intention. There are the hypothesis:

H6.Experience may impact farmers' information usage intention positively with the medium of perceived usefulness.

H7.Experience may impact farmers' information usage intention positively with the medium of perceived ease of use.

\subsection{Information searching and learning motivation}

Earlier studies suggested that intensive usage motivation would act on the adoption(Lee et al.,2005). We argue that farmers who exhibit higher levels of information searching and learning motivation will be more interested in agriculture information than those with lower levels.

H8.Searching intention is related to perceived usefulness positively.

H9.Learning intention is related to perceived usefulness positively.

H10.Searching intention is related to perceived ease of use positively.

H11.Learning intention is related to perceived ease of use positively. 


\subsection{Perceived risk}

According to consumer behavior theory, the risk that people consider subjectively is not the really exiting one.When deciding whether to adopt information, farmers' perceived risk is crucial. The more likely famrers are to do something venturesome, the more intensive the usage intention is.In this paper, we define farmers' perceived risk as risk preference and the ability to bear risk. It is supposed that perceived risk and risk assuming ability influence intention through information attitude.

H12.Risk preference is related to farmers' information attitude.

H13.Risk assuming ability is related to farmers' information attitude.

\subsection{Individual variables}

The function of information is recessive and indirect,both of which determine the discrepant outcomes when adopted by kinds of profiles of users.For example,it may be easier to catch on the deeply signification of information for farmers with the higher education. In the same way ,those that have taken on the larger plowland may be more willingly to adopt information.In this paragraph, age, income,education and plowland acreage are included as individual variables.

\section{ANALYSIS}

\subsection{Data collection}

Data in the study were collected with a sample of two hundred and thirtyone farmers from thirteen different areas in China. The items were measured on a 5 -point Likert-type scale ( $1=$ strongly disagree to $5=$ strongly agree).

\subsection{The analysis of the factors}

\subsubsection{The original factors}

Correlation analysis is used in this study. The original TAM model shows a well applicability in the study. Perceived usefulness of information directly affects its intention to use, with a coefficient of 0.501.Perceived ease of use exhibits a positive relationship on perceived usefulness (.581), which means 
if farmers consider that information technology is easy to master, the usage intention is strong. Besides, there exits a relationship between intention to use and perceived usefulness via attitude, either is perceived ease of use. Attitude influences the usage intention significantly, with a coefficient of .710. Results are shown in Figure 3.

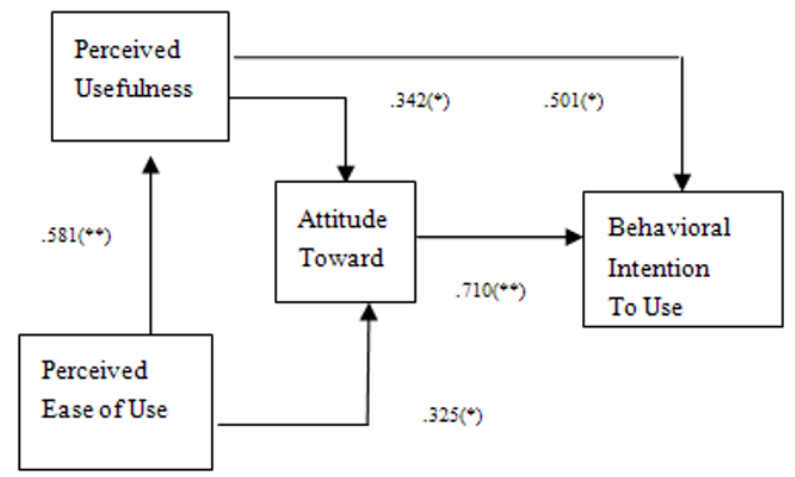

Fig.3: The correlation between factors of the original model

\subsubsection{The extended factors}

It is indicated that experience is directly associated with perceived usefulness, and more strongly associated with perceived ease of use (.538).Usually, if farmers have contacted with information and received some help, they are willing to consider it useful and the intention to use is stronger. Moreover, experience reduces the difficulty to master information, which in turn enhances farmers' confidence in information usage.

In this paper, information motivation is defined as information searching and learning intention. It is showed that searching intention has impact on perceived usefulness and ease of use positively, but not intensively. This phenomenon could be explained that although farmers are willing to search information voluntarily, they do not know how to do. The correlation both between learning intention and perceived usefulness on one hand and between learning intention and ease of use on the other hand are great, with coefficients of .518 and .631. The relationship is presented in Table 1. 
Table 1: The correlation coefficients between the factors

\begin{tabular}{ccccc}
\hline \multicolumn{2}{c}{ External variable } & $\begin{array}{c}\text { Perceived } \\
\text { usefulness }\end{array}$ & $\begin{array}{c}\text { Perceived } \\
\text { ease of use }\end{array}$ & Attitude \\
\hline \multicolumn{2}{c}{ Experience in information } & $.357\left(^{*}\right)$ & $.538(* *)$ & \\
\hline $\begin{array}{c}\text { Information } \\
\text { motivation }\end{array}$ & $\begin{array}{c}\text { Searching intention } \\
\text { Learning intention }\end{array}$ & .133 & .271 & \\
Perceived risk & Risk preference & $.518\left(^{* *}\right)$ & $.631(* *)$ & \\
& Risk assuming ability & & & $.433\left(^{*}\right)$ \\
\hline
\end{tabular}

Perceived risk is referred to validate whether it affects farmers' information usage intention via attitude in this study. The result also yields the evidence that perceived risk influences attitude positively, and risk preference has stronger impact on attitude than risk assuming ability.

As for the individual variables, it is showed that education and income affect farmers' usage intention positively, but not so strongly as we have forecast before.

\section{DISCUSSION AND CONCLUTIONS}

This paper aimed to exam farmers' information usage intention in China based on the technology acceptance model (TAM) added with new variables. TAM model has so far mainly been used to explain the technology adoption topic. The results suggest that the application of the TAM model is helpful in obtaining a better understanding of factors influencing farmers' information usage intention.

It is showed that farmers' attitude and perceived usefulness both directly drive usage intention. The support is found that perceived usefulness and perceived ease of use determine attitude toward agriculture information usage, and perceived ease of use also has impact on perceived usefulness.

The findings highlight that experience in information usage and learning intention are paramount predictors of perceived usefulness and ease of use. Risk preference is positively correlative with attitude. The impact of searching intention is not evident, neither is assuming ability. Our findings also demonstrate that except for education and income, the farmer individual variables such as age and plowland acreage do not have the evident effect.

Based on the conclusions, some suggestions are put forth here:

Varying the categories of information.

To make full use of information, different kinds of farmers could be supplied with various information. Take risk preference for example, on the 
one hand, farmers who always avoid to do something venturesome may be interested in market information and product information, on the other hand, the ones that are likely to try something new may also concern about the advanced technologies or investment information.

Making the channels of information transmission convenient for farmers.

Even though having known about the importance of agriculture information gradually, most farmers are short of the ability to search for information effectively. To simplify the information transmission methods may lead to information more reachable.

Paying attention to farmers who have experience in information usage. The research shows that farmers are likely to accept agriculture information if they have the similar experience before, moreover, their confidence and behavior will influence others with no experience positively.

\section{LIMITATIONS AND FUTURE RESEARCH}

Several key findings emerged from this study, and the TAM model has been proved to be an appropriate frame to examine information adoption .However, the number of the sample of farmers is small in the survey, which may have a negative influence on the conclusions.

Various types of agriculture information will appear up in the information market, and some kinds of the information may have the attributes of the private product. Future research could be focused on farmers' willingness to pay for information in order to know about farmers' information demand and adoption deeply.

\section{ACKNOWLEDGEMENTS}

This study was supported by the National Science \& Technology Support Program (2006BAD10A07-02). The authors are grateful to the farmers that took part in the investigation.

\section{REFERENCES}

Fred D.Davis,Richard P.Bagozzi, Paul R.Warshaw.User Acceptance of Computer Technology: A Comparison Of Two Theoretical Models, Management Science,1989,35:982-1003

Fred D.Davis.Perceived Usefulness, Perceived Ease of Use, and User Acceptance of Information Technology, IS Quarterly, 1989, 9:319-340 
Hyun-Hwa Lee,Ann Marie Fiore,Jihyun Kim.The role of the technology acceptance model in explaining effects of image interactivity technology on consumer responses, International Journal of Retail \& Distribution Management,2006,8:621-644

J.Alberto Castaneda, Francisco Munoz-Leiva, Teodoro Luque.Web Acceptance Model(WAM):Moderating effects of user experience, Information \& Management,2007,44:384-396

Juan Carlos Roca,Chao-Min Chiu,Francisco Jose Martinez.Understanding e-learning continuance intention:An extension of the Technology Acceptance Model, HumanComputer Studies,2006,64:683-696

Mattew K.O.Lee,Christy M.K,Cheung,Zhaohui Chen.Acceptance of Internet-based learning medium:the role of extrinsic and intrinsic motivation, Information \& Management,2005,42:1095-1104

Sally McKechnie, Heidi Winklhofer, Christine Ennew. Applying the technology acceptance model to the online retailing of financial services, International Journal of Retail \& Distribution Management, 2006, 34:388-410

William R.King, Jun He.A meta-analysis of the technology acceptance model, Information \& Management, 2006, 43:740-755

Yi-Shun Wang,Hsiu-Yuan Wang,Daniel Y.Shee.Measuring e-learning systems success in an organizational context:Scale development and validation, Computers in Human Behavior,2007,23:1792-1808 